\title{
Prótese fixa sobre dentes e implantes: relato de caso
}

\author{
Fixed prosthesis on teeth and implants: a case report \\ Prótesis fija sobre dientes e implantes: reporte de caso
}

Recebido: 02/09/2021 | Revisado: 12/09/2021 | Aceito: 16/09/2021 | Publicado: 17/09/2021

\author{
Ana Paula de Almeida Gomes \\ ORCID: https://orcid.org/0000-0001-8581-3358 \\ Centro Universitário de Volta Redonda, Brasil \\ E-mail: anapaulaliz.almeida@outlook.com \\ Camila Gobbi de Carvalho Barbosa \\ ORCID: https://orcid.org/0000-0001-9825-2449 \\ Centro Universitário de Volta Redonda, Brasil \\ E-mail: camila-gobbi@hotmail.com \\ Cláudio Luís de Melo-Silva \\ ORCID: https://orcid.org/0000-0001-5093-2189 \\ Centro Universitário de Volta Redonda, Brasil \\ E-mail: claudiolmelo@hotmail.com \\ Tereza Cristina Favieri de Silva-Melo \\ ORCID: https://orcid.org/0000-0001-5309-7190 \\ Centro Universitário de Volta Redonda, Brasil \\ E-mail: terezafavieri@gmail.com \\ Rodrigo Xavier de Freitas \\ ORCID: https://orcid.org/0000-0001-6444-4792 \\ Centro Universitário de Volta Redonda, Brasil \\ E-mail: rodrigoxavierdefreitas@gmail.com \\ Cristiane Fonseca de Carvalho \\ ORCID: https://orcid.org/0000-0001-6302-127X \\ Centro Universitário de Volta Redonda, Brasil \\ E-mail: crisfoncar@hotmail.com \\ Roanna de Castro Teixeira \\ ORCID: https://orcid.org/0000-0001-7828-8057 \\ Centro Universitário de Volta Redonda, Brasil \\ E-mail: roannadecastro@ hotmail.com
}

\begin{abstract}
Resumo
Diversos fatores podem ocasionar perdas dentárias no indivíduo, prejudicando a harmonia facial e a fisiologia do sistema estomatognático, influenciando principalmente na mastigação, na fonação e no equilíbrio articular. Em casos mais complexos que envolvem perdas dentárias múltiplas, é necessário o restabelecimento da dimensão vertical de oclusão (DVO), assim como a procurapela estética e aumento da autoestima é constantemente requisitada. O objetivo deste trabalho foi relatar um caso clínico de reabilitação oral com prótese implanto suportada associada à prótese dento suportada, em que foram restabelecidos a DVO, estética, função e equilíbrio do sistema estomatognático. O tratamento do paciente de 56 anos, leucoderma e do gênero masculino, foi realizado na clínica odontológica do Centro Universitário de Volta Redonda e após anamnese, exame clínico, análise das fotografias, da panorâmica e dos modelos de estudo observou- se perda de função mastigatória, estética e colapso na dimensão vertical de oclusão. O paciente negou alergias e relatou ser diabético e hipertenso, porém em tratamento controlando as morbidades. A reabilitação oral foi realizada através da confecção de prótese fixa tipo protocolo Branemark anterior modificado sobre dentes e implantes. Conclui-se que a satisfação do paciente se fez muito evidente com a melhora funcional e estética através da instalação da prótese provisória tipo protocolo anterior modificado a partir de resina acrílica PMMA, respondendo de maneira satisfatória a todos os requisitos necessários até essa fase da reabilitação, restabelecendo a dimensão vertical de oclusão, devolvendo a eficiência mastigatória, assim como reequilibrando o sistema estomatognático influenciando psicossocialmente em sua qualidade de vida.
\end{abstract}

Palavras-chave: Reabilitação bucal; Dimensão vertical; Estética dentária.

\section{Abstract}

Several factors can cause tooth loss in the individual, impairing facial harmony and the physiology of the stomatognathic system, influencing mainly on chewing, phonation and joint balance. In more complex cases involving multiple tooth losses, it is necessary to reestablish the vertical dimension of occlusion (VDO), as well as the search for aesthetics and increased self-esteem are constantly required. The objective of this work will be to report a clinical case of oral rehabilitation with implant supported prosthesis associated with dental supported prosthesis, in 
which the VDO, aesthetics, function and balance of the stomatognathic system will be restored. The treatment of the 56 years old, leucoderma, malepatient, was carried out at the dental clinic of the University Center of Volta Redonda and after anamnesis, clinical examination, analysis of thephotographs, panoramic radiography and the study models, it was observed loss of masticatory function, aesthetics and collapse in the vertical dimension of occlusion. The patient denied allergies and reported being diabetic and hypertensive, but under treatment to control these morbidities. Oral rehabilitation was performed through the confection of a modified Branemark protocol fixed prosthesis over teeth and implants. It is concluded that patient satisfaction was evident with the improvement of functional and aesthetic by the installation of the provisional prosthesis modified protocol from PMMA acrylic resin, satisfactorily answering all the necessary requirements until this rehabilitation phase, restoring the vertical dimension of occlusion, returning masticatory efficiency, as well as rebalancing the stomatognathic system psychosocially influencing his quality of life. Keywords: Mouth rehabilitation; Vertical dimension; Esthetics dental.

\section{Resumen}

Son varios los factores que pueden provocar la pérdida de dientes en los individuos, afectando a la armonía facial y a la fisiología del sistema estomatognático, influyendo principalmente en la masticación, la fonación y el equilibrio articular. En los casos más complejos que implican la pérdida de múltiples dientes, es necesario restablecer la dimensión vertical de la oclusión (dvo), y la búsqueda de la estética y el aumento de la autoestima se solicita constantemente. El objetivo de este estudio fue reportar un caso clínico de rehabilitación oral con prótesis soportada por implantes asociada a una prótesis soportada por dentadura, en la que se restauró la ddo, la estética, la función y el equilibrio del sistema estomatognático. El tratamiento del paciente de 56 años, varón de raza caucásica, se realizó en la clínica dental del centro universitario de volta redonda y tras la anamnesis, examen clínico, análisis de fotografías, panorámicas y modelos de estudio se observó una pérdida de la función masticatoria, estética y colapso en la dimensión vertical de la oclusión. La paciente negó ser alérgica y dijo ser diabética e hipertensa, pero estar en tratamiento para controlar estas morbilidades. La rehabilitación oral se realizó mediante la confección de prótesis fijas tipo protocolo branemark anterior modificado sobre dientes e implantes. Concluimos que la satisfacción del paciente fue muy evidente con la mejora funcional y estética a través de la instalación de la prótesis provisional tipo protocolo anterior modificado de resina acrílica pmma, respondiendo satisfactoriamente a todos los requerimientos necesarios hasta esta fase de la rehabilitación, restaurando la dimensión vertical de la oclusión, devolviendo la eficiencia masticatoria, así como reequilibrando el sistema estomatognático influyendo psicosocialmente en su calidad de vida.

Palabras clave: Rehabilitación bucal; Dimensión vertical; Estética dental.

\section{Introdução}

A qualidade de vida do indivíduo é diretamente relacionada a uma condição de saúde oral adequada, visto que, as funções das estruturas quando realizadas corretamente oferecem benefícios para a saúde geral do paciente, diferentemente de quando os componentes do sistema são danificados ocorrendo perda de função (Carvalho, Melo, Ramos, Lima \& Carvalho, 2019). Traumas, cáries, alterações periodontais e patologias podem estar associados à perda dentária que causará ao paciente alterações na fisiologia do sistema estomatognático desde distúrbios na mastigação até desordens articulares (Haddad, Pellizzer, Mazaro, Verri \& Falcón-Antenucci, 2008).

Devido à desarmonia facial ocasionada pelo edentulismo, a procura pela estética e aumento da autoestima é constantemente requisitada por esses pacientes (Castro, Corrêa, Costa, Martins \& Silva, 2020). Portanto o equilíbrio entre a estética vermelha que engloba lábios, mucosa e gengiva, incluindo o contorno do zênite gengival, ea estética branca que nada mais é do que os elementos dentários, garantema recomposição do equilíbrio do sorriso e da harmonia facial implicando, de forma positiva, na autoimagem do paciente (Quesada, Rizzardi, Franciscatto \& Arrais, 2014; Cardozo, 2017).

A perda total ou parcial de elementos dentários compromete, além da estética, a harmonia na dimensão vertical de oclusão (DVO) do paciente, que é definida como a altura do terço inferior da face quando as superfícies oclusais estão em contato. Sendo assim, o restabelecimento da DVO também é de extrema importância para o funcionamento correto do sistema estomatognático e para o sucesso do tratamento reabilitador (Cézar \& Silva 2019).

Inúmeras são as possibilidades de procedimentos para reabilitação oral desses pacientes, dentre elas pode-se citar próteses provisórias, prótese total ou parcial removível, próteses fixas que podem ser dento suportada ou implanto suportada e ainda a associação das técnicas (Dantas, 2012; Oliveira, 2018). 
Para pacientes com perdas dentárias totais ou parciais pode-se optar pela utilização de próteses fixas implanto suportadas que são, na maioria das vezes, a primeira opção de escolha visto que, proporcionam maior eficácia da função mastigatória, maior conforto e diminui o reparo e manutenção (Rocha, Souza, Fernandes, Garcia \& Zavanelli, 2013). Entretanto, quando há opção de associar o uso de implantes com prótese dento suportada a distribuição de forças ocorre de forma mais equilibrada uma vez que, diferente dos implantes, os dentes possuem ligamento periodontal que é responsável pela propriocepção, fazendo com que as forças mastigatórias sejam mantidas dentro dos limites fisiológicos (Misch \& Bidez, 2019; Silva, 2020).

É importante ressaltar que os estudos sobre implantes foram introduzidos por Branemark em meados de $1960 \mathrm{em}$ que contemplou o potencial de cicatrização, remodelação e a interação osso, medula e sangue definindo assim o princípio de osseointegração que seria o contato íntimo entre osso alveolar do paciente e o implante colocado (Oliveira, 2018).

O uso de implantes osseointegrados promove aos pacientes além da estabilidade oclusal a promoção da harmonia facial, garantindo um bom prognóstico desde que todo o protocolo seja seguido corretamente (Faverani et al., 2011) por meio de exames pré-operatórios, elaboração de um plano de tratamento correto envolvendo saúde dos tecidos periodontais, sobrecarga oclusal, diâmetro e distribuição dos implantes na arcada, hábito e história médica do paciente (Quesada et al., 2014; Romanos, 2017).

O objetivo deste trabalho foi relatar um caso clínico de reabilitação oral com prótese implanto suportada associada à prótese dento suportada, em que serão restabelecidos a DVO, estética, função e equilíbrio do sistema estomatognático.

\section{Metodologia}

O presente estudo refere-se a um relato de caso, caracterizado como um estudo exploratório, descritivo e qualitativo (Pereira et al., 2018). Foram seguidos os princípios éticos de pesquisa envolvendo seres humanos, sendo submetido ao Comitê de Ética em Pesquisa do Centro Universitário de Volta Redonda (CoEPs) - UniFOA com número CAAE 34490920.7.0000.5237 e aprovado em 12 de julho de 2020.

\section{Relato de Caso}

Paciente P.R.G. de 56 anos, gênero masculino, leucoderma, compareceu a clínica odontológica do Centro Universitário de Volta Redonda (UniFOA) no dia 31 de maio de 2019, queixando da estética e da dificuldade de alimentar-se.

Para a realização do exame clínico e um planejamento adequado, foram solicitadas fotografias intraorais (Figuras 1 a 3) e radiografia panorâmica (Figura 4). 
Figura 1: Oclusão intraoral anterior.

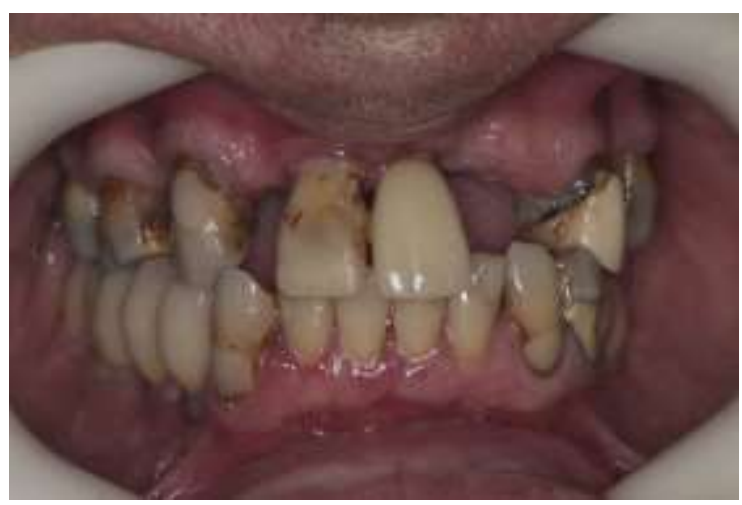

Fonte: Autores.

Figura 2: Oclusão intraoral direita.

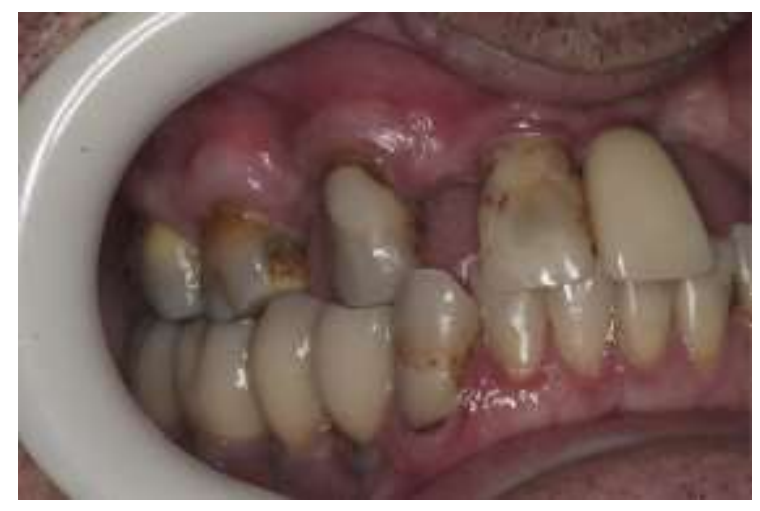

Fonte: Autores.
Figura 3: Oclusão intraoral esquerda.

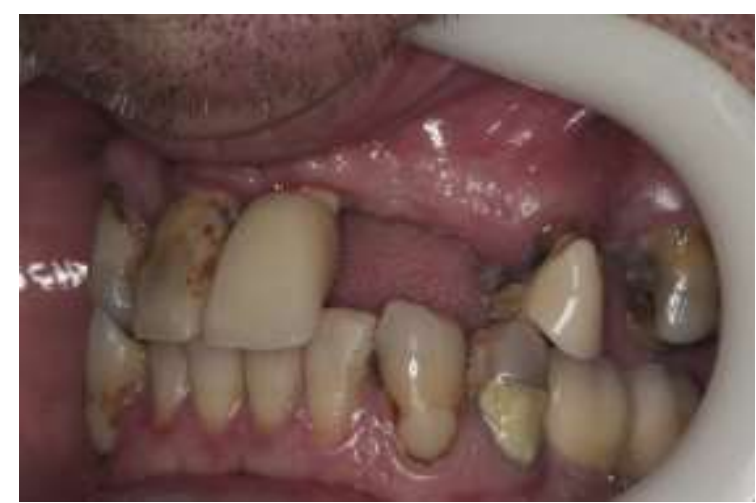

Fonte: Autores.

Figura 4: Radiografia panorâmica inicial.

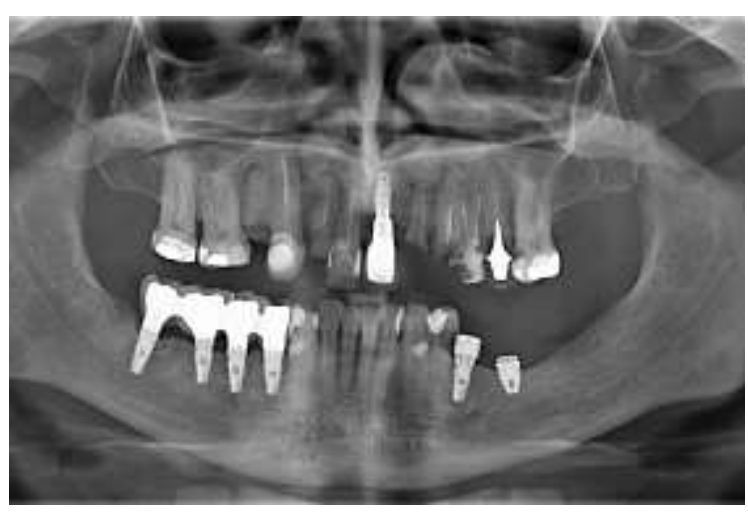

Fonte: Autores.

O paciente não relatou dor ou desconforto na articulação têmporo-mandibular (ATM), mas a aparência facial do paciente mostrou sinais de colapso na dimensão vertical de oclusão (DVO) (Figura 5), significando que o sistema estomatognático se adaptou a esta diminuição gradual do terço inferior da face, uma vez que há oclusão do hemi-arco direito envolvendo os implantes e os elementos dentários. 
Figura 5: Fotografia frontal inicial.

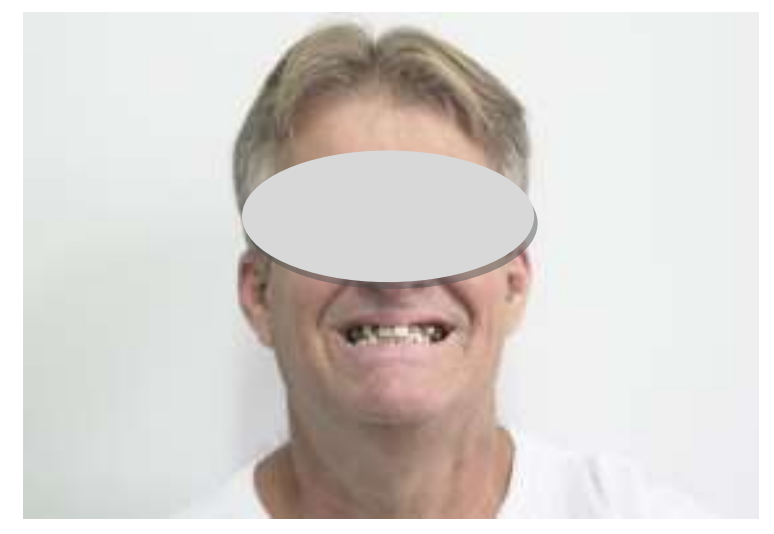

Fonte: Autores.

O plano de tratamento foi realizado conforme as seguintes etapas:Prótese parcial removível provisória em resina acrílica:

- Exodontia dos elementos 12, 14, 22, 23 e 25;

- Instalação dos implantes na região dos elementos 12, 14, 23 e 25;

- Reabertura dos implantes;

- Confecção do protocolo anterior modificado provisório de resina acrílica;

- Reanatomização dos molares superiores e dos elementos anteriores inferiores com resina composta;

O procedimento foi iniciado a partir da moldagem das arcadas para a confecção da prótese parcial removível provisória em resina acrílica para restabelecer a estética dos remanescentes radiculares que serão extraídos.

Iniciaram-se as extrações dos restos radiculares dos elementos 12 e 14 e adaptação da prótese parcial removível (Figura 6).

Figura 6: Adaptação da prótese parcial removível.

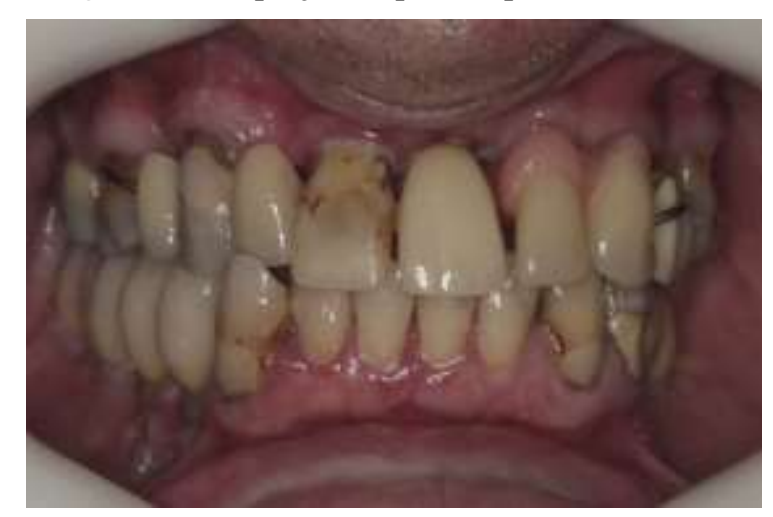

Fonte: Autores.

A seguir, prosseguiram as extrações dos remanescentes dos elementos 22,23 e 25 de maneira atraumática para preservar o contorno das papilas gengivais e o osso alveolar. 
Aguardou-se o tempo de cicatrização de 90 dias para a cirurgia de instalação dos implantes osseointegrados na região dos elementos $12,14,23$ e 25 .

Após 120 dias (4 meses), foi realizada nova radiografia panorâmica (Figura 7) onde nenhuma alteração patológica foi observada nos sítios dos implantes e realizou-se a reabertura e colocação de cicatrizadores.

Figura 7: Radiografia panorâmica com os implantes instalados.

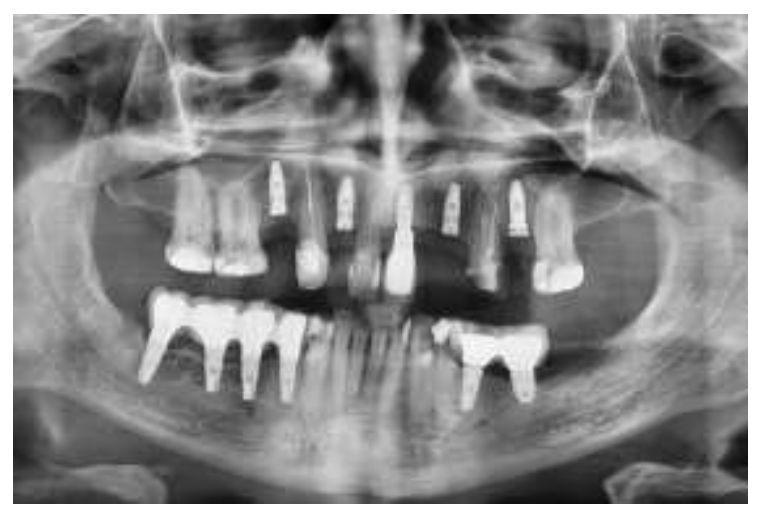

Fonte: Autores.

Foram realizadas moldagens das arcadas superior e inferior utilizando silicona de adição base pesada e leve Adsil (ColteneVigodent) para confecção dos modelos de trabalho.

Para determinação do espaço inter oclusal e registro de mordida foi construído um Jig de Lucia, utilizando resina acrílica Pattern Duralay (Reliance) e silicona de adição.

Os modelos articulados foram enviados ao laboratório de prótese panfecção da prótese provisória fixa tipo protocolo Branemark anterior modificado em resina acrílica polimetilmetacrilato (PMMA- CAD CAM) e enceramento diagnóstico dos molares superiores segundo a nova DVO.

Foram realizadas as exodontias dos elementos 11, 13 e 24 com posterior colocação da prótese de transição fixa através da instalação dos minis pilares sobre os implantes com torque de 25N, e os cilindros provisórios em titânio (Figura 8) foram parafusados sobre os minis pilares com torque de $15 \mathrm{~N}$, marcados e cortados com disco de carborundum na altura referente à prótese, que foi desgastada na região dos cilindros e adaptada a eles por meio de resina acrílica.

Figura 8: Cilindros provisórios instalados

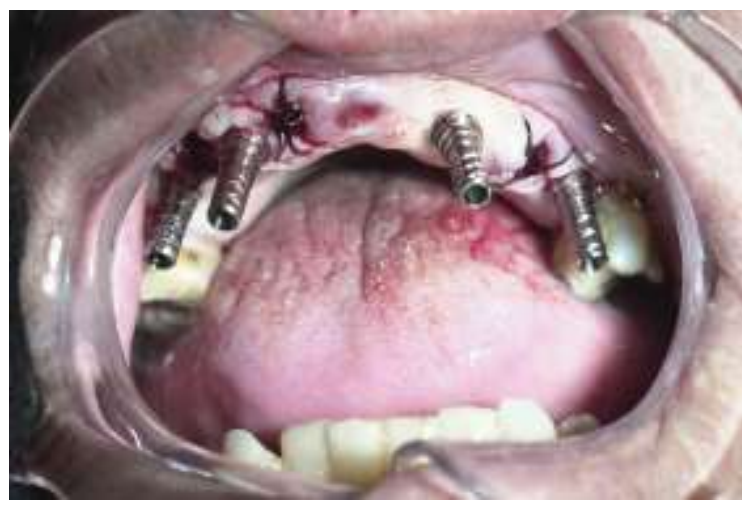

Fonte: Autores. 
Foi feito o acabamento, registro de oclusão e a prótese de resina PMMA foi parafusada sobre os minis pilares, repondo todos os elementos da região do 14 ao 25 (Figura 9).

Figura 9: Prótese tipo protocolo anterior modificada instalada.

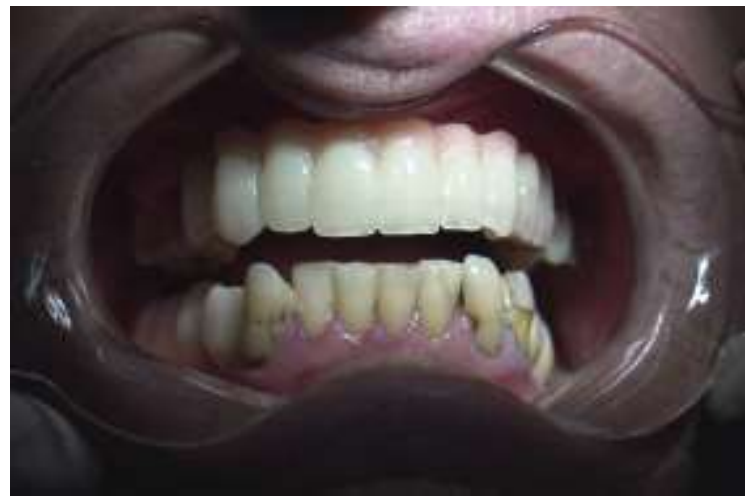

Fonte: Autores.

Após 7 dias e com a remoção das suturas das exodontias dos elementos 11, 13 e 24, foi realizada a reanatomização dos molares superiores $(16,17$ e 26) com resina composta, tendo como referência o enceramento diagnóstico previamente executado no modelo, utilizando guias de silicone incolor (Zhermack- elite transparent)para que a passagem de luz do fotopolimerizador fosse possível para correta reprodução da anatomia oclusal, restabelecendo o equilíbrio da oclusão do paciente.

Em sessões subsequentes foi realizada a troca das restaurações dos elementos 32 e 42, e reanatomização dos elementos 31, 32, 33, 34, 41, 42 e 43. Posteriormente foi feito acabamento e polimento das restaurações.

A reabilitação realizada no paciente atingiu todas as expectativas, devolvendo DVO, função, estética e equilíbrio do sistema estomatognático (Figuras 10 e 11).

Figura 10: Oclusão anterior pós tratamento.

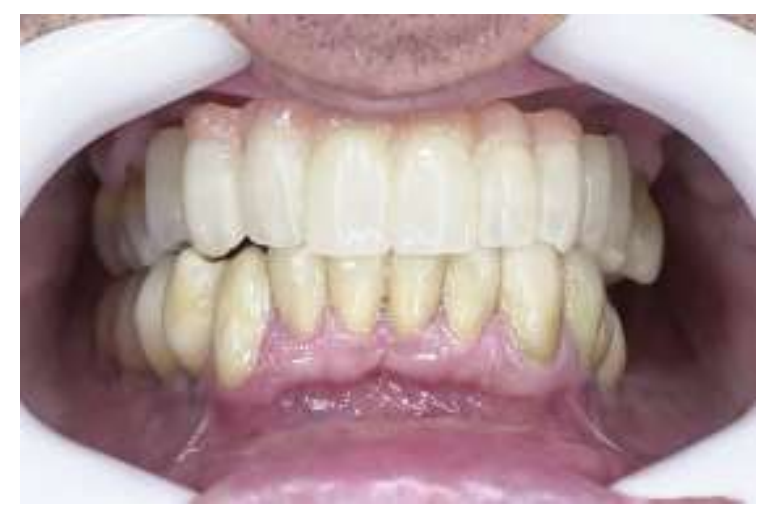

Fonte: Autores.
Figura11: Fotografia frontal final.

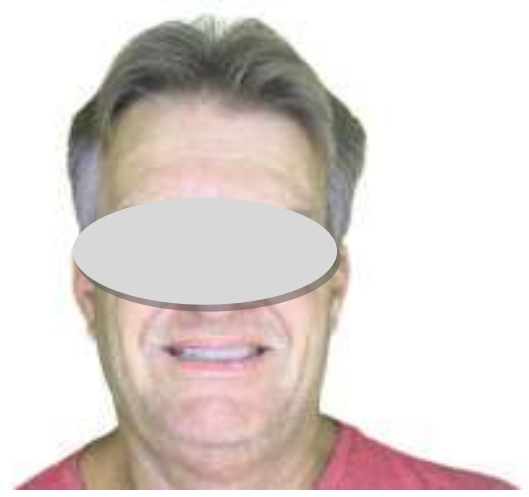

Fonte: Autores.

O caso clínico encontra-se em fase de finalização em que o paciente passará por período de transição de 6 meses com a prótese de transição fixa, para posterior confecção da prótese definitiva metaloplástica com barra metálica fundida concomitantemente com a adaptação da placa miorrelaxante. 


\section{Discussão}

Um indivíduo saudável é aquele que apresenta, além da ausência de doença, condições de bem estar social com um equilíbrio de fatores psicológicos e sociais, estabelecendo uma harmonia no meio em que se vive. A odontologia participa desse equilíbrio através da manutenção do sistema estomatognático (SE) que está diretamente relacionado com a preservação e reabilitação das funções de fala, mastigação, deglutição,assim como da estética (Fernandes Neto, Neves \& Simamoto Junior, 2013), mantendo dessa forma a fisiologia e harmonia entre todos os componentes anatômicos do SE, ou seja, sem causar danos patológicos a essas estruturas (Cardoso, 2003; Cavalcanti, Oliveira \& Batista, 2015).

A perda dentária causará ao paciente alterações na fisiologia do sistema estomatognático desde distúrbios na mastigação, desarmonia na dimensão vertical de oclusão, até desordens articulares (Haddad et al., 2008 \& Carvalho et al., 2019). Sendo assim, a qualidade de vida do indivíduo é diretamente relacionada a uma condição de saúde oral adequada, visto que, as funções das estruturas quando realizadas corretamente oferecem benefícios para a saúde geral do paciente, diferentemente de quando os componentes do sistema são danificados ocorrendo perda de função (Cézar \& Silva, 2019; Dantas, 2012). A aparência facial do paciente relatado apresentou sinais de colapso da dimensão vertical devido às perdas dentárias e demonstrou características de aprofundamento do sulco nasogeniano e curvatura de lábio invertida.

Levando em consideração a desarmonia facial causada pelo edentulismo, um planejamento protético deve envolver um plano de tratamento adequado permitindo uma melhor previsibilidade dos resultados finais. Para isso, deve-se documentar a maior quantidade de dados sobre o paciente, analisando-o como um todo, através de uma anamnese detalhada, exame clínico completo, exames complementares, modelos de estudo de qualidade e fotografias intra e extraorais (Vidotti, Lanza \& Pergoraro, 2014; Moreira Junior et al., 2018).

Para a reparação e substituição de elementos perdidos existem as próteses provisórias, próteses totais (dentaduras), próteses fixas e próteses parciais removíveis que serão escolhidas visando um melhor prognóstico de reabilitação oral para o paciente (Dantas, 2008; Oliveira, 2014). Porém no presente estudo foram utilizadas a prótese parcial removível provisória no período de transição das extrações iniciais e prótese parcial fixa provisória após as extrações múltiplas, para equilíbrio do sistema estomatognático e para posterior colocação da prótese parcial fixa.

Ressaltando a importância da prótese provisória, durante o período de cicatrização das extrações realizadas para a futura colocação dos implantes, o paciente necessita de uma prótese de transição para que as funções mastigatórias, fonéticas e estéticas não sejam prejudicadas; alémde adaptar o paciente ao resultado final de todo o processo e proteger a mucosa em cicatrização (Cho, Shetty, Froum, Elian \& Tarnow, 2017; Teixeira, Valente, Castro, Morelli \& Reis, 2019).

Diante da situação encontrada no paciente, optou-se pela confecção da prótese provisória fixa sobre implantes após a realização de todas as extrações planejadas. A prótese fixa é uma proposta reabilitadora permanente às estruturas remanescentes cujo objetivo está relacionado à substituição de um único elemento ou até mesmo uma reabilitação total (Vidotti et al., 2014), com a finalidade de devolver função mastigatória, promover maior conforto e diminuir o reparo e manutenção (Rocha et al., 2013).

Para um resultado de excelência em casos reabilitadores com prótesefixa, além de um correto planejamento, é necessário que haja o contato íntimo entre o osso alveolar do paciente e o implante colocado, denominado osseointegração, processo com ausência de sintomas onde há fixação rígida e estabilidade no osso durante aplicação de cargas funcionais (Oliveira, 2014; Albrektsson, Branemark, Hansson \& Lindström, 1981; Branemark, 2005; Medeiros, 2017). 
Tal condição foi observada no caso relatado depois de 120 dias após a colocação dos implantes, em que por meio de nova radiografia panorâmica observou-se características de nível ósseo satisfatórias indicando aosseointegração dos implantes, confirmada através da reabertura dos mesmos, garantindo a estabilidade e permitindo o sucesso do tratamento a longo prazo.

Desde 1969 a reabilitação com implantes osseointegrados tem demonstrado bons resultados e pode ser realizada de duas maneiras distintas: através de carga imediata em que a instalação da prótese ocorre em média de 72 horas após as exodontias dos elementos, simplificando o procedimento e reduzindo o período de cicatrização; ou tardia, que consiste na instalação dos implantes após o período de 2 a 3 meses de cicatrização antes que a carga funcional seja aplicada (Misch \& Scortecci, 2006; Canellas, Medeiros, Figueredo, Fischer \& Ritto, 2019). No trabalho realizado optouse pela carga tardia, devido às condições sistêmicas do paciente e condições anatômicas dos tecidos periodontais que apresentavam sinais de inflamação.

Vários fatores estão relacionados com o sucesso desse tipo de reabilitação oral, dentre eles podemos citar as propriedades físico-químicas da superfície que atuam na interface osso implante e a saúde dos tecidos peri-implantares que deverão ser analisados tridimensionalmente antes da colocação do implante (Faverani et al., 2011; Bottino, Itinoche \& Guimarães, 2007). Além disso,para a longevidade do tratamentodeve-se considerar a sobrecarga oclusal, diâmetro, textura e localização do implante, hábitos e história médica do paciente (Quesada et al., 2014; Romanos, 2017).

Apesar dos altos índices de sucesso, a reabilitação com implantes gera questionamentos no que diz respeito à biomecânica, distribuição de forças nas estruturas de suporte e propriocepção do paciente. Devido à ausência de ligamento periodontal, a dissipação de forças no implante difere do dente uma vez que no elemento dentário a percepção dolorosa é rápida e aguda, desencadeando um mecanismo de proteção através de sinais clínicos e radiográficos (Misch \& Bidez, 2006; Silva, 2020; Dominici, 2019).

Para tratamento utilizando prótese tipo protocolo clássico onde envolve reabilitação total, são utilizados de 6 a 8 implantes na maxila (Dominici, 2019). Entretanto, ao analisar o perímetro do arco do paciente em questão, foi planejada a reabilitação da prótese tipo protocolo modificado utilizando 4 implantes distribuídos na região de segundo pré molar a segundo pré molar, devido à presença dos molares em boas condições contra indicando a exodontia, garantindo propriocepção e melhor distribuição de forças nas estruturas de suporte. Dessa forma, foi possível a instalação da prótese provisória tipo protocolo anterior modificado, sem barra metálica a fim de lapidar as relações oclusais antes da confecção da prótese definitiva.

A população edêntula sofre reflexos negativos na autoestima com a perda dentária devido à diminuição da estética e origem de alterações psicológicas. Porser dependente de fatores socioeconômicos, a estética influencia diretamente na vida dos indivíduos, pois sua autoimagem irá refletir em seu comportamento em sociedade e nas relações interpessoais (Haddad et al., 2008; Barreto et al., 2019; Scrascia et al., 2020).

Para que isso não ocorra, as próteses tanto provisórias como definitivas têm por função devolver harmonia e equilíbrio ao sistema estomatognático (Texeira et al., 2019), assim como no caso relatado em que por meio da utilização da prótese provisória fixa, devolveu ao paciente função mastigatória, suporte labial, harmonia da relação dente-gengiva através da gengiva artificial da prótese, equilíbrio muscular e recuperação da simetria da face. Dessa forma, as expectativas do paciente foram superadas, melhorando sua qualidade de vida fisicamente e psicologicamente.

É importante ressaltar que o paciente deve ser responsável, além da boa higiene, por cuidados com a mastigação de certos alimentos que por ventura esteja acostumado a ingerir e pelo controle de hábitos parafuncionais que podem gerar falhas no tratamento protético (Vidotti et al., 2014; Livio, Silvia \& Poluha, 2019). A colaboração e o comprometimento do paciente relatado foram essenciais para o sucesso do tratamento reabilitador até o momento. 


\section{Conclusão}

Com base no planejamento proposto em que foi utilizada a prótese fixa sobre dentes e implantes foi possível restabelecer a dimensão verticalde oclusão do paciente, devolver a eficiência mastigatória, assim comoreequilibrar o sistema estomatognático.

O material utilizado para a confecção da prótese provisória tipo protocolo anterior modificado (resina acrílica PMMA - CAD CAM) respondeude maneira satisfatória a todos os requisitos necessários até essa fase da reabilitação, diminuindo consideravelmente o custo, protegendo os tecidos durante a cicatrização e preparando o paciente para a execução do procedimento reabilitador definitivo.

A satisfação do paciente se fez muito evidente com a melhora funcional e estética influenciando psicossocialmente em sua qualidade de vida, visto que múltiplas perdas dentárias afetam, além das condições funcionais, a sua autoestima.

Para trabalhos futuros, sugere-se que sejam realizados estudos relacionados às propriedades mecânicas e estéticas da resina PMMA para o sucesso e longevidade das reabilitações utilizando esse material.

\section{Referências}

Albrektsson, T., Branemark, P. I., Hansson, H. A., Lindström, J. (1981). Osseointegrated titanium implants. Requirements for ensuring a long-lasting direct bone-to-implant anchorage in man. Acta Orthopaedica Scandinavica, 52(2), 155-170.

Barreto, J. O., Sousa, M. L. A., Silva-Júnior, S. E., Freire, J. C. P., Araújo, T. N., Freitas, G. B., \& Dias-Ribeiro, E. (2019). Impactos psicossociais da estética dentária na qualidade de vida de pacientes submetidos a próteses: revisão de literatura. Archives of Health Investigation, 8(1), 48-52.

Bottino, M. A., Itinoche, M. K., \& Guimarães, M. V. M. (2007). Inter-relação periodontia- implante-prótese. In: E. Saba-Chujfi, \& S. A. Santos-Pereira. Periodontologia integração e resultados. Artes Médicas

Branemark, P. I. (2005) The osseointegration book. From calvarium to calcaneus. Batavia: Quintessence Books.

Canellas, J. V. S., Medeiros, P. J. D., Figueredo, C. M. S., Fischer, R. G., \& Ritto, F. G. (2019). Which is the best choice after tooth extraction, immediate implant placement or delayed placement with alveolar ridge preservation? A systematic review and meta-analysis. Journal of Cranio-Maxillofacial Surgery, 47 (11), 1793-1802.

Cardoso, A. C. (2003). Oclusão Pra Você e Pra Mim. Livraria Santos.

Cardozo, T. F. (2017). Otimização da Estética Vermelha e Branca: Revisão de Literatura. Monografia, Universidade do Sul de Santa Catarina, Tubarão, Santa Catarina, Brasil.

Carvalho, L. F., Melo, J. R. O., Ramos, J. G., Lima, R. A., \& Carvalho, F. A. A. (2019). O impacto do edentulismo na qualidade de vida de pacientes edêntulos. Revista da AcBO, 8(1), 40-48.

Castro, G. Á. L., Corrêa, P. F., Costa, M. D. M. A., Martins, L. H. B., Silva, G. R., \& Dietrich, L. (2020). Reabilitação anterior de maxila com implantes osseointegrados: da prótese parcial removível a prótese parcial fixa. Revista de Odontologia Contemporânea, 4(1), 1-10.

Cavalcanti, Y. W., Oliveira, L. M. C., \& Batista, A. U. D. (2015). Prótese Parcial Removível Provisória Tipo Overlay na Reabilitação Oral de Paciente com Colapso Oclusal Posterior. Revista Brasileira de Ciências da Saúde, 19(2), 143-150.

Cézar, H. F., \& Silva, F. B. (2019). Recuperação da Dimensão Vertical de Oclusão com Prótese Temporária Overlay: Relato de Caso. Archives of Health Investigation, 8(6), 290-295.

Cho, S. C., Shetty, S., Froum, S., Elian, N., \& Tarnow, D. (2017). Fixed and removable provisional options for patients undergoing implant treatment. Compendium of Continuing Education in Dentistry, 28(11), 604-609.

Dantas, E. M. (2012). A Importância do Restabelecimento da Dimensão Vertical de Oclusão na Reabilitação Protética. Revista Odonto, 20(40), 41-48.

Dominici, F. F. C. S. (2019). Uma abordagem clínica sobre prótese total sobre implante. Monografia, Faculdade de Sete Lagoas, São Luís, Brasil.

Faverani, L. P., Ramalho-Ferreira, G., Gaetti-Jardim, E. C., Okamoto, R., Shinohara, E. H., Assunção, W. G., \& Garcia Junior, I. R. (2011). Implantes Osseointegrados: Evolução e Sucesso. Revista Salusvita, 30(1),47-58.

Fernandes Neto, A. J., Neves, F. D., \& Simamoto Junior, P. C. (2013). Oclusão. Artes Médicas.

Haddad, M. F., Pellizzer, E. P., Mazaro, J. V. Q., Verri, F. R., \& Falcón-Antenucci, R. M. (2008). Conceitos Básicos para a Reabilitação Oral por meio de Implantes Osseointegrados - Parte I: Influência do Diâmetro e do Comprimento. Revista Odontológica de Araçatuba, 29(1), 30-37. 
Livio, E. M., Silva, R. S., \& Poluha, R. L. (2019). Higienização e manutenção de prótese total fixam sobre implante. Archives of Health Investigation, 8(7), 390-393.

Medeiros, F. L. A. (2017) Osseointegração de Implantes Dentários em Pacientes Diabéticos: Uma Revisão Integrativa da Literatura Científica. Monografia, Universidade Federal do Rio Grande do Norte, Natal, RN, Brasil.

Misch, C. E., \& Bidez, M. W. (2006). Considerações Oclusais para a Prótese Implantossuportada: Oclusão Implantoprotegida. In: C. E. Misch (Ed.). Prótese sobre Implantes (Ed. 2, pp. 472-510). São Paulo: Santos.

Misch, C. E., Scortecci, G. M. (2006). Carga Imediata: Aplicações em Implantodontia. In: C. E. Misch (Ed.). Prótese sobre Implantes (Ed. 1, pp. 531-567). São Paulo: Santos.

Moreira Junior, R., Ribeiro, P. D., Condezo, A. F. B., Cini, M. A., Antoni, C. C., \& Moreira, R. (2018). Fundamentos da análise facial para harmonização estética na odontologia brasileira. Clínica e Pesquisa em Odontologia, 9(1), 59-65.

Oliveira, A. S. (2019). Técnicas em próteses dentárias: noções básicas, classificação e confecção. Érica.

Pereira, A. S., Shitsuka, D. M., Parreira, F. J., \& Shitsuka, R. (2018). Metodologia da pesquisa científica. UFSM. https://repositorio.ufsm.br/bitstream/handl e/1/15824/Lic Computacao_Metodologia-Pesquisa-Cientifica.pdf?sequence=1.

Quesada, G. A. T., Rizzardi, M., Franciscatto, L. J., \& Arrais, F. R. (2014). Condicionamento gengival visando o perfil de emergência em prótese sobre implante. Saúde (Santa Maria), 40(2), 9-18.

Rocha, S. S., Souza, D. R., Fernandes, J. M. A., Garcia, R. R., \& Zavanelli, R. A. (2013). Próteses Totais Fixas Tipo Protocolo Bimaxilares. Revista Odontológica do Brasil Central, 21(60), 21-27.

Romanos, G. E. (2017). Estratégias de Preservação Tecidual para Favorecer a Estabilidade a Longo Prazo dos Tecidos Moles e Duros. The International Journal of Periodontics and Restorative Dentistry, 2(1), 89-97.

Scrascia, R., Fiorillo, L., Gaita, V., Secondo, L., Nicita, F., \& Cervino, G. (2020) Implant-Supported Prosthesis for Edentulous Patient Rehabilitation. From Temporary Prosthesis to Definitive with a New Protocol: A Single Case Report. Prosthesis, 2(1), 10-24.

Silva, R. F. (2020). Prótese sobre implante cimentada x parafusada: uma revisão narrativa da literatura. Dissertação de Mestrado, Universidade Católica Portuguesa, Viseu, Portugal.

Teixeira, A. B. V., Valente, M. L. C., Castro, D. T., Morelli, V. G., \& Reis, A. C. (2019). Prótese de transição x prótese sobre implantes: relato de caso. Revista Odontológica do Brasil Central, 28(84), 30-34.

Vidotti, H. A., Lanza, M. D. S., \& Pegoraro, L. F. (2014). Planejamento em prótese parcial fixa. In: L. F. Pegoraro, C. E. Rezende, C.O. Cunha, F. H. Stancari, H. A. Vidotti, L. A. Santos, et al. Fundamentos de Prótese Fixa (Ed. 1, pp. 34-42). Artes Médicas. 